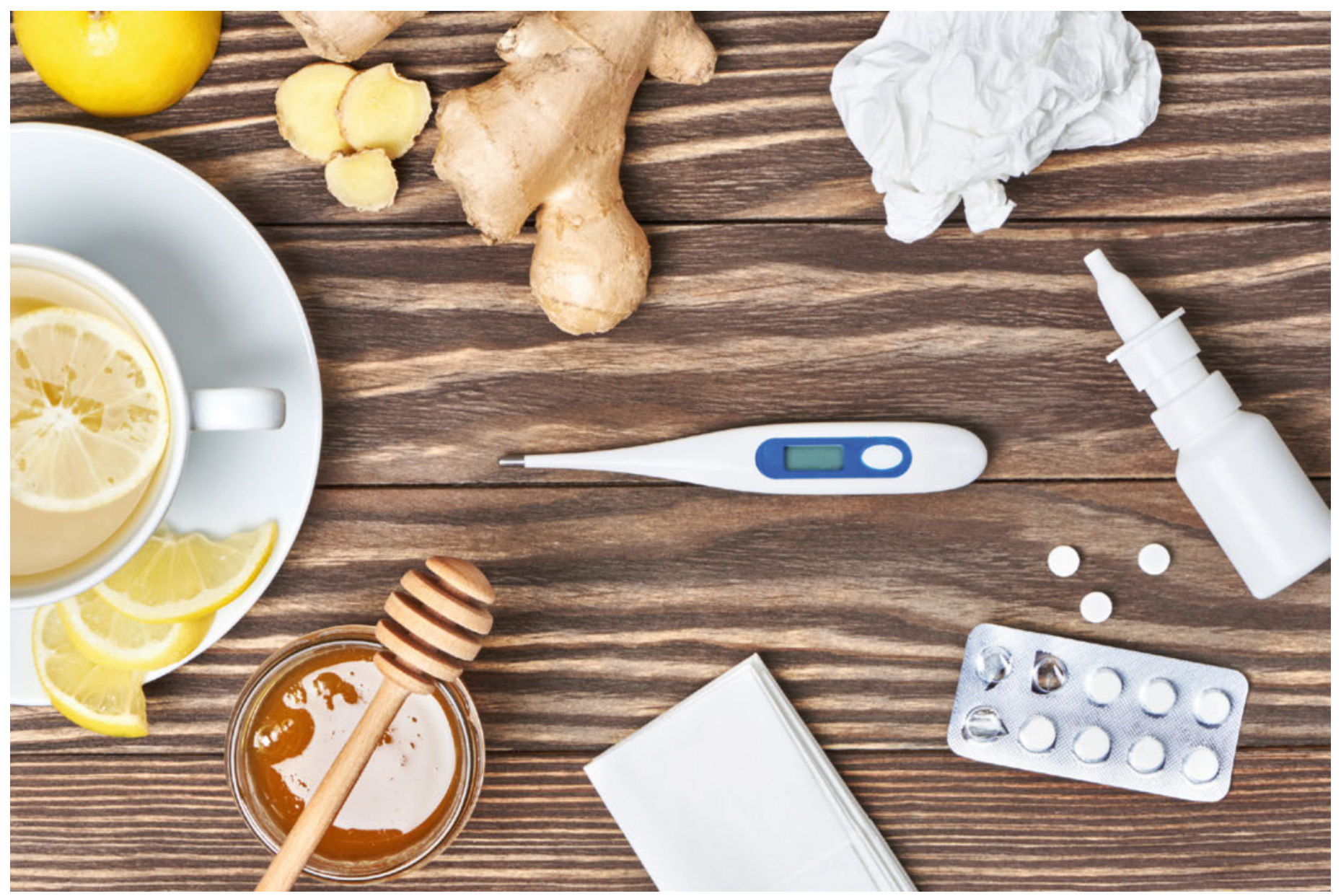

\title{
Pharmas, médecine - des questions que les gens posent
}

\section{Jean Martin}

Dr méd., membre de la rédaction

Bonjour Jean,

Je me tourne vers toi à propos de médecine. Je suis entourée de gens qui critiquent l'industrie pharmaceutique. Ils sont assez radicaux et certains, corps et âme, affirment que, tout comme l'industrie agro-alimentaire est une guerre contre la nature, les médicaments représentent une guerre contre ton corps. On peut selon eux régler les problèmes en donnant beaucoup d'attention à son corps et son esprit, à l'écoute de ses besoins - gérer le stress, avoir une alimentation de produits bio non transformés. Un ami aurait guéri d'un cancer en changeant radicalement son hygiène de vie - processus qui lui a demandé beaucoup de temps et des décisions fortes: s'occuper de lui-même était un défi à temps plein durant cette maladie.

J'écoute les avis et fais mes expériences. Les petites maladies qui traversent ma vie sont rares mais j'ai remarqué que certaines façons de faire pouvaient influer beaucoup. Depuis un an, si j'ai un rhume par exemple, je lui donne de l'attention, bois des bouillons, tisanes et beaucoup d'eau, prends le temps de me reposer. Et j'ai l'impression de guérir beaucoup plus 
vite que quand, dans ces mêmes circonstances de rhume, je l'ignorais et continuais ma vie comme si de rien n'était.

\section{Et ma réponse:}

Chère F.,

Merci pour ce mail intéressant. S'agissant de tes questions sur l'industrie pharmaceutique, il faut distinguer plusieurs choses. D’abord, le côté qu'on peut dire négatif: clairement cette industrie fait partie de l'économie à but lucratif et elle se comporte comme les marchands de voitures, de voyages, de produits de luxe, de canons, etc. Les pharmas développent ce qui permet de gagner de l'argent et de rémunérer ses actionnaires. Ce qui explique que, sous réserve de rares exceptions, les pharmas ne consacrent guère d'argent à rechercher des traitements de maladies fréquentes - et graves - dans les pays pauvres, là il n'y aurait pas assez d'acheteurs solvables pour des produits efficaces.

C'est pour cela que la lutte et la prévention d'affections qui font des centaines de millions de malades chroniques, et beaucoup de morts, mais qui touchent peu le Nord, ont avancé lentement. On pense au paludisme (malaria), à la tuberculose (qui a pratiquement disparu de nos pays), à des parasitoses et autres maladies exotiques, souvent invalidantes. Par contre, on peut noter que pour le VIH/sida apparu dans les années 1980, beaucoup plus d'efforts ont été consentis, parce que le sida a touché de manière importante, dans les pays développés aussi, certains groupes. Groupes qui ont efficacement sensibilisé les milieux politiques, économiques et professionnels concernés de manière à obtenir des financements conséquents, pour la recherche et pour les soins.

Cette «négligence» des problèmes des régions pauvres est infiniment regrettable... On peut parler de grave manque d'éthique sociale mais c'est la logique qui continue à prévaloir. Idéalement, dans ce domaine et d'autres, un impératif de responsabilité planétaire (y compris écologique et climatique) devrait être intégré par les acteurs de l'économie comme par les pouvoirs publics et la société civile.

Autre point: oui, on peut dire que les médicaments sont "contre nature». En un sens, toute la médecine cherche à éviter ou guérir ou soulager les atteintes dues à la nature. Si on laissait notre organisme exposé à tout ce que «propose» notre milieu de vie, peut-être serait-ce magnifique du point de vue de tes camarades, mais nous serions, en moyenne, en bien mauvaise santé et vivrions beaucoup moins longtemps. Il y aurait énormément de "casse» prématurée suite aux infections, accidents, aux grossesses et accouchements difficiles, aux décès de petits enfants etc. Effectivement, d'une certaine manière, la médecine lutte contre l'environnement qui ne nous veut pas toujours du bien, il s'en faut de beaucoup.

Un chiffre: ces dernières décennies, l'espérance de vie dans les pays industrialisés a augmenté d'une année tous les quatre ans; par rapport à il y a quarante ans, la population vit dix ans de plus en moyenne! Ce n'est pas dû uniquement à la médecine mais elle a joué là un rôle notable. Impressionnant, non?

Où je ne suis pas du tout d'accord, c'est à propos d'une prétendue non-efficacité des médicaments. Il y a des produits dits "de confort» dont on pourrait se passer, c'est vrai. Sauf exception, il est inutile de prendre des suppléments vitaminiques par exemple. Les pharmas font certaines recherches sur des créneaux où il y a de l'argent à gagner mais pas vraiment de gains significatifs de santé à espérer (je l'ai dit... l'économie n'est pas tenue de s'orienter vers des buts d'intérêt général). Par contre, des quantités de médicaments sont efficaces et utiles, les antibiotiques parmi d'autres (même si on les utilise aujourd'hui de manière excessive et que certaines bactéries deviennent résistantes à tous). Les médicaments ont apporté aussi de grandes contributions aux progrès ("merveilleux", on pourrait utiliser ce mot) dans les soins intensifs, la chirurgie moderne et le traitement des cancers, entre autres.

Enfin: Magnifique ce que tu me dis de l'écoute de ton propre corps, de toi-même. Continue à t'intéresser à ce qui permet sans médecine de garder un bon équilibre de vie.

Cela étant, si tes amis ou toi deviez souffrir d'une affection grave ou que vous vous cassiez en morceaux dans un accident, je serais heureux que vous consentiez à voir (aussi) mes confrères orthodoxes. Enfin et de plus, les médecins d'aujourd'hui devraient accepter de bonne grâce que leurs malades fassent appel à la médecine occidentale et à des techniques complémentaires qui ont d'autres fondements ou origines (encore qu'il soit vrai que tous n'acceptent pas encore cette attitude des patients et le dialogue avec eux sur ces sujets).

Dr Jean Martin

Crédit photo

@ Ryzhov $\mid$ Dreamstime.com 